\title{
The Versatile Array of Neutron Detectors at Low Energy (VANDLE)
}

\author{
Catalin Matei ${ }^{1 *}$, D.W. Bardayan ${ }^{2}$, J.C. Blackmon ${ }^{3}$, J.A. Cizewski ${ }^{4}$, R.K. Grzywacz ${ }^{5}$, \\ S.N. Liddick ${ }^{5}$, W.A. Peters ${ }^{4}$, F. Sarazin ${ }^{6}$ \\ ${ }^{1}$ Oak Ridge Associated Universities, Oak Ridge, TN 37831 USA \\ 2 Physics Division, Oak Ridge National Laboratory, Oak Ridge, TN 37831 USA \\ ${ }^{3}$ Department of Physics and Astronomy, Louisiana State University, Baton Rouge, LA 70803 \\ ${ }^{4}$ Department of Physics and Astronomy, Rutgers University, New Brunswick, NJ 08903 USA \\ ${ }^{5}$ Department of Physics and Astronomy, University of Tennessee, Knoxville, TN 37996 USA \\ ${ }^{6}$ Department of Physics, Colorado School of Mines, Golden, CO 80401 USA
}

The Versatile Array of Neutron Detectors at Low Energy (VANDLE) is a new array of plastic scintillator bars under development for measurements at the Holifield Radioactive Ion Beam Facility at Oak Ridge National Laboratory. The array is highly modular allowing the configuration of the individual elements to be optimized for particular experimental requirements. Proposed experiments include $(\mathrm{d}, \mathrm{n})$ reactions and beta-delayed neutron emission studies relevant to nuclear astrophysics.

10th Symposium on Nuclei in the Cosmos

July 27 - August 12008

Mackinac Island, Michigan, USA

*Speaker. E-mail: mateic@ornl.gov 


\section{Introduction}

The Versatile Array of Neutron Detectors at Low Energy (VANDLE) is under development at the Center for Radioactive Ion Beam Studies for Stewardship Science to detect low-energy neutrons in the $100 \mathrm{keV}$ to $10 \mathrm{MeV}$ range. The array will be a powerful new tool for studying the structure of exotic nuclei with low-energy radioactive ion beams available from the Holifield Radioactive Ion Beam Facility (HRIBF) at Oak Ridge National Laboratory (ORNL).

The proposed experimental program includes studies with $(\mathrm{d}, \mathrm{n})$ reactions on neutron- and proton-rich nuclei and measurements of the beta-delayed neutron decay strength in nuclei near the neutron drip-line. On the proton-rich side studies of the $(\mathrm{d}, \mathrm{n})$ reaction on ${ }^{56} \mathrm{Ni}$ [1] and ${ }^{25} \mathrm{Al}$ [沟] are relevant for nuclear astrophysics. The doubly-magic ${ }^{56} \mathrm{Ni}$ is a waiting point in the rp-process, which occurs at the high temperatures and densities present in x-ray bursts and x-ray pulsars. A measurement of the ${ }^{2} \mathrm{H}\left({ }^{56} \mathrm{Ni}, \mathrm{n}\right){ }^{57} \mathrm{Cu}$ reaction cross section to populate the astrophysically important 1/2- level near $1106 \mathrm{keV}$ would allow a first measurement of its spectroscopic factor and thus the first direct determination of $\Gamma_{p}$ and the ${ }^{56} \mathrm{Ni}(\mathrm{p}, \gamma)^{57} \mathrm{Cu}$ reaction rate. Beta decay properties of neutron-rich nuclei are among the most important quantities for the modeling of the rapid neutron capture process. Investigation of nuclei near ${ }^{78} \mathrm{Ni}$ and ${ }^{132} \mathrm{Sn}$ would determine beta-delayed neutron decay lifetimes and branching ratios which can be used directly in r-process calculations [3].

The investigation of $(\mathrm{d}, \mathrm{n})$ reactions and beta-delayed neutron emission imposes specific requirements on the properties of the neutron detector array. One challenge in these measurements is the detection of the reaction neutrons with good energy and angular resolution. Experiments are complicated by reactions in inverse kinematics with relatively low beam intensities which require large solid angle coverage and high efficiency.

In this paper we present the design of the Versatile Array of Neutron Detectors at Low Energy (VANDLE). The array is modular which allows the configuration to be optimized for particular experimental requirements. We propose one configuration optimized for studies of $(\mathrm{d}, \mathrm{n})$ reactions in inverse kinematics using radioactive ion beams at energies near the Coulomb barrier and one optimized for beta-delayed neutron emission studies.

\section{Detector Design}

The most important technical factors that have to be considered when designing a neutron array are the detection efficiency, energy resolution, angular resolution, detection threshold and cross talk between detector elements. The energy resolution is limited by the detector thickness because the neutron can interact anywhere within the detector volume. Thus, good time-of-flight measurements require rather thin detectors. On the other hand, good detection efficiency requires covering a large solid angle with thicker detectors to increase the neutron's probability of interaction. Achieving good angular resolution requires higher segmentation of the array with the disadvantage of increasing the cross-talk. There are trade-offs between these factors, and designing the array for a larger number of different experiments requires some of these factors to have a higher priority than others. The design of the neutron array is based on plastic scintillator bars viewed at opposing ends by photomultiplier tubes (PMTs). Smaller detector bars provide better time-of-flight resolution and better sensitivity to lower energy neutrons but have reduced efficiency to higher energy neutrons. 
Longer, thicker detector bars cover a larger solid angle with good intrinsic detection efficiency but have to be placed further away from the target to achieve good energy resolution.

\subsection{Configuration for (d,n) studies}

The cross section for the ${ }^{2} \mathrm{H}\left({ }^{56} \mathrm{Ni}, \mathrm{n}\right){ }^{57} \mathrm{Cu}$ reaction is largest and most indicative of the angular momentum transfer of the populated state at forward center-of-mass angles $\theta_{c m}=5-60^{\circ}$, corresponding to laboratory angles of $\theta_{l a b}=75-160^{\circ}$. To accurately determine the excitation energy for a state populated in the $(\mathrm{d}, \mathrm{n})$ reaction, the neutron energy, as well as the reaction angle, must be accurately measured. The position within a bar at which a neutron interacts can be localized from the relative timing between the two PMTs. A more effective arrangement for the study of $(\mathrm{d}, \mathrm{n})$ transfer reactions would be obtained by orienting the scintillator bars perpendicular to the incident beam direction such that each bar subtends close to a unique laboratory angle. One possible configuration that might be used for studies of $(\mathrm{d}, \mathrm{n})$ reactions is shown in Fig. 1 .

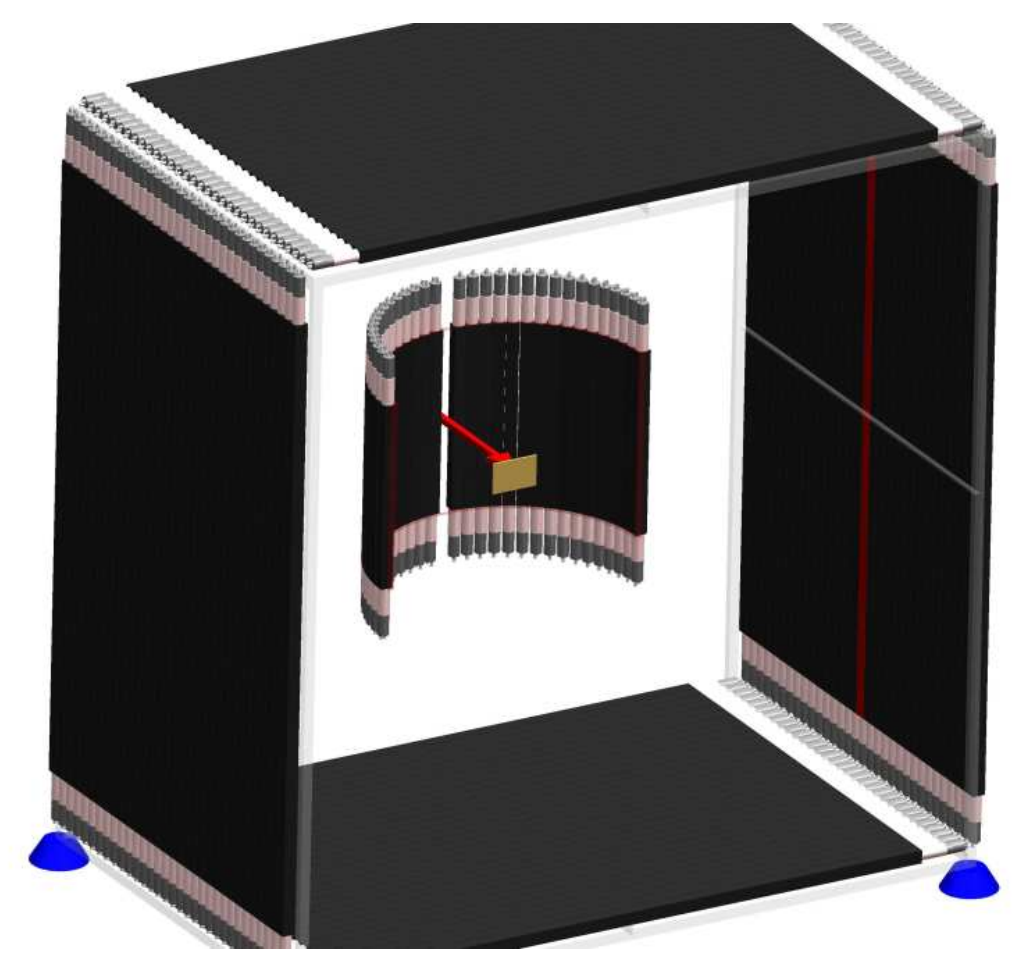

Figure 1: Computer-drafted image of the (d,n) configuration for VANDLE, including 128 bars $(5 \times 5 \times 200$ $\left.\mathrm{cm}^{3}\right)$ arranged in 4 walls and 50 bars $\left(2.9 \times 2.9 \times 60 \mathrm{~cm}^{3}\right)$ arranged in a $80-\mathrm{cm}$ diameter half barrel.

Four walls are constructed using 32 bars each of the $5 \times 5 \times 200-\mathrm{cm}^{3}$ scintillator that make 4 sides of a square as viewed from the beam direction. The walls are positioned $1.5 \mathrm{~m}$ from the target position, with the bars oriented perpendicular to the incident beam direction. With this arrangement, scattering angles of $\theta_{l a b}=62-118^{\circ}$ are subtended by the walls, and the laboratory angle can be determined to a precision of $1^{\circ}$. We find that the simulated detection efficiency is relatively constant as a function of the laboratory angle. The laboratory neutron energy resolution is determined in this configuration to a precision of about $4 \%$. 
The neutron energies at more backward laboratory angles are lower, and thus the use of the smaller scintillator bars is advantageous for increased light collection. We propose to subtend backward laboratory angles using $502.9 \times 2.9 \times 60-\mathrm{cm}^{3}$ scintillator bars in a half-barrel arrangement. The combination of good laboratory angle determination, good neutron energy resolution, and relatively high efficiency makes this configuration well suited for measurements of $(\mathrm{d}, \mathrm{n})$ reactions.

\subsection{Configuration for beta-n studies}

One possible delayed neutron decay setup is shown in Fig. 2, a potentially excellent tool to realize the systematic measurement of the beta decay strength distribution in the region near ${ }^{78} \mathrm{Ni}$ and ${ }^{132} \mathrm{Sn}$.

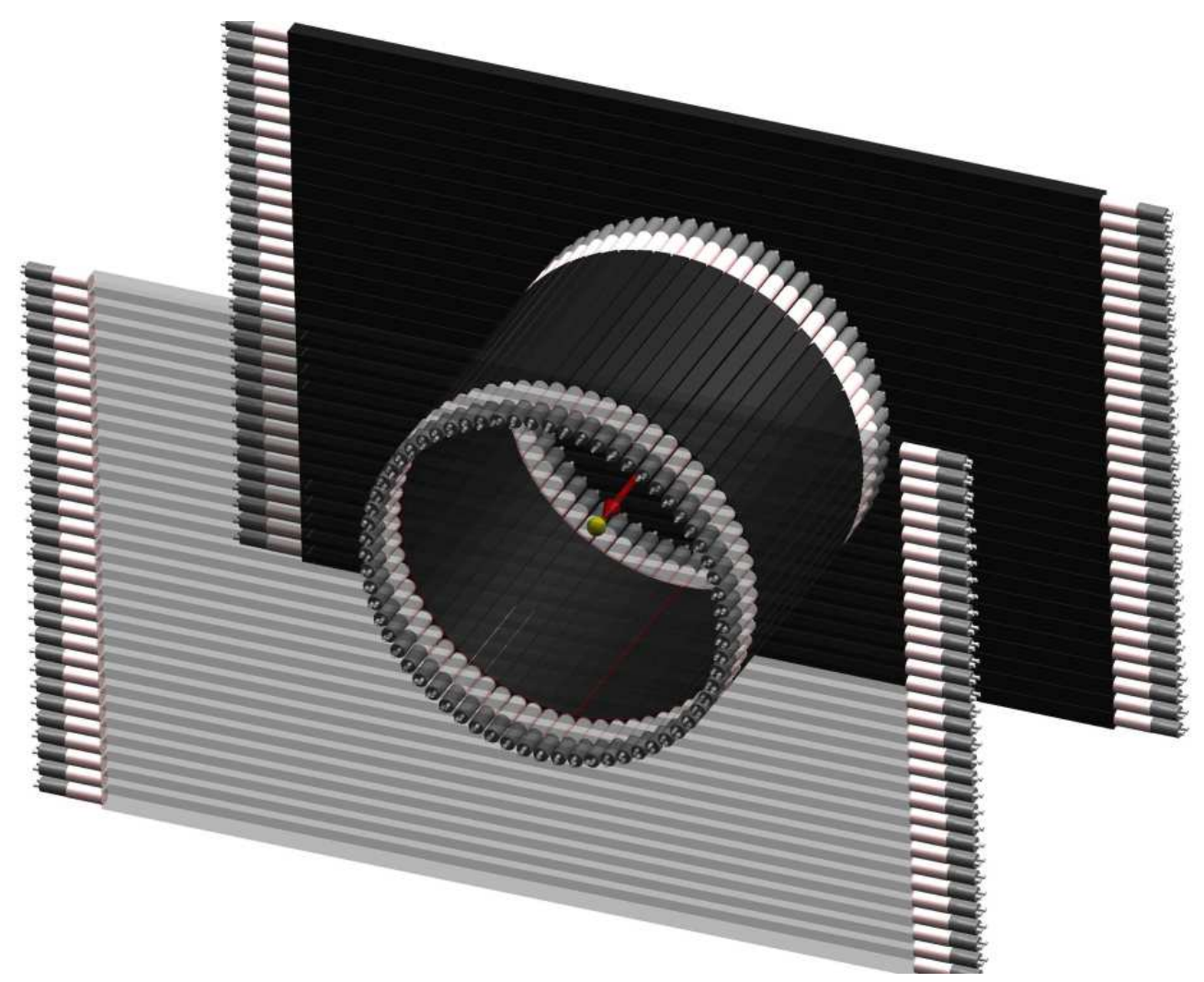

Figure 2: Computer-drafted image of the beta-delayed neutron studies configuration for VANDLE, including 100 bars $\left(2.9 \times 2.9 \times 60 \mathrm{~cm}^{3}\right)$ arranged in a $80-\mathrm{cm}$ diameter barrel and 64 bars $\left(5 \times 5 \times 200 \mathrm{~cm}^{3}\right)$ arranged in 2 walls closing the barrel and giving a geometrical coverage of near $100 \%$.

This configuration deploys 100 bars of the $2.9 \times 2.9 \times 60-\mathrm{cm}^{3}$ scintillator arranged in a barrel configuration surrounding the point of implantation. The radius of the barrel with all 100 bars is 40 $\mathrm{cm}$, providing a geometrical coverage of about $30 \%$ of $4-\pi \mathrm{sr}$, with a low threshold and relatively high efficiency for detection of low energy neutrons. Two walls of the 5-cm bars are constructed at forward and backward angles to close the ends of the barrel and increase the geometrical coverage to essentially $100 \%$ of $4-\pi$ sr. As shown, 32 bars of $5 \mathrm{~cm} \times 5 \mathrm{~cm}$ scintillator are used in each wall at a distance of $75 \mathrm{~cm}$ from the point of implantation. 
The larger scintillator bars do have reduced sensitivity to the lowest energy neutrons, but they provide a higher efficiency for higher energy neutrons and a better energy resolution from time-offlight. The combination of the barrel of smaller detectors at a close distance and the two walls of larger detectors provides relatively efficient neutron detection across a broad range of energies.

\section{Summary}

A new neutron detector array VANDLE for measuring low-energy neutrons in the $100 \mathrm{keV}$ to $10 \mathrm{MeV}$ range with good energy resolution and high efficiency is proposed for use at HRIBF at Oak Ridge National Laboratory. Detector prototypes are currently being assembled and tested. The scientific program includes $(\mathrm{d}, \mathrm{n})$ reaction measurements and beta-delayed neutron emission studies important for nuclear structure physics and astrophysics.

\section{Acknowledgments}

This work is supported in part by the U.S. Department of Energy under contract numbers DE-FG52-03NA00143 (ORAU, Rutgers, and UTK) and DE-AC05-00OR22725 (ORNL).

\section{References}

[1] O. Forstner, H. Herndl, H. Oberhummer, H. Schatz, and B.A. Brown, Phys. Rev. C 64, 045801 (2001).

[2] J. Jose, A. Coc, and M. Hernanz, ApJ 520, 347 (1999).

[3] K. Langanke and M. Wiescher, Rep. Prog. Phys. 64, 1657 (2001).

[4] LAND Collaboration, Nucl. Instr. Meth. A 314, 136(1992).

[5] MONA Collaboration, Nucl. Instr. Meth. A 543, 517(2005). 